\title{
Comorbilidad psicopatológica en obesidad
}

\section{Psychopathological comorbidity in obesity}

\author{
J.I. Baile, M.J. González
}

\section{RESUMEN}

La obesidad es uno de los problemas de salud más importantes a comienzos del siglo XXI, afectando a millones de personas en todo el mundo, siendo una las principales causas de mortandad. En la actualidad no está clara la relación entre obesidad y psicopatología, y tampoco se ha aclarado si las posibles alteraciones psicológicas son causa o consecuencia de la obesidad. El presente trabajo tiene por objeto revisar algunas de las aportaciones recientes (2007-2010) relativas a la comorbilidad psicopatológica y la obesidad. De dicho análisis se deriva que existen ciertas alteraciones psicológicas que parecen ser más comunes en las personas obesas, como pueden ser la depresión, la ansiedad, el consumo de sustancias, etc., aunque no queda clara la dirección de la relación explicativa; así mismo, también existen resultados contradictorios. La constancia de cierta probabilidad de comorbilidad psicopatológica obliga al clínico a considerar su evaluación en los casos de obesidad y, previsiblemente, a incluir un plan de intervención terapéutico de orden psicológico-psiquiátrico.

Palabras clave. Obesidad. Sobrepeso. Comorbilidad. Psicopatología.

\begin{abstract}
Obesity is one of the most important health problems in the early twenty-first century as it affects millions of people around the world and remains a major cause of mortality. At present there is no clear link between obesity and psychopathology, and whether the possible psychological disorders are a cause or a consequence of obesity has not yet been clarified either. This paper reviews some recent contributions (20072010) related to the comorbidity between psychopathology and obesity. It can be concluded from this analysis that certain psychological disorders seem to be more common in obese people, such as depression, anxiety, substance abuse, etc., although the direction of the explanatory relation is not clear; moreover, the opposite result has also been found. The evidence of likely comorbid psychopathology means that the practitioner must consider its assessment in obesity cases, and include a plan for a psychiatric and/or psychological therapeutic intervention.
\end{abstract}

Keywords. Obesity. Overweight. Comorbidity. Psychopathology.
Departamento de Psicología. Universidad a Distancia de Madrid.

Recepción: 25 de febrero de 2011

Aceptación provisional: 6 de abril de 2011

Aceptación definitiva: 19 de abril de 2011

\section{Correspondencia}

Dr. José I. Baile

Departamento de Psicología

Universidad a Distancia de Madrid

Camino de la Fonda, $\mathrm{n}^{\circ} 20$

28400 Collado Villalba. Madrid

e-mail: ignaciobaile@hotmail.com 


\section{INTRODUCCIÓN}

En la actualidad, la obesidad se define como un exceso de grasa ${ }^{1}$ y la forma más aceptada para su valoración es el Índice de Masa Corporal (IMC) o Índice de Quetelet, que se obtiene dividiendo el peso en kilogramos por la altura en metros al cuadrado $\left(\mathrm{kg} / \mathrm{m}^{2}\right)$. Se considera que una persona tiene sobrepeso si su IMC se encuentra entre 25 y 30 , y obesidad cuando es superior a 30 , siendo más grave cuanto mayor sea dicho índice ${ }^{2}$. En niños y adolescentes se puede utilizar el mismo índice, si bien los valores de referencia para determinar el sobrepeso u obesidad son específicos para cada edad, por lo que deben consultarse tablas de crecimiento específicas.

La obesidad es uno de los principales problemas de salud a nivel mundial en la actualidad. Por un lado, debido a sus cifras de prevalencia: según la Organización Mundial de la Salud (OMS) ${ }^{3}$, en el año 2015 se esperan aproximadamente 2.300 millones de adultos con sobrepeso y más de 700 millones con obesidad, por lo que se le denomina "la epidemia del siglo XXI". Por otro lado, por sus elevados índices de comorbilidad con enfermedades de índole tanto física como psicológica. Respecto de los primeros, la obesidad se relaciona con numerosas alteraciones biomédicas, especialmente con los trastornos cardiovasculares.

Se ha comprobado que a mayor IMC se produce mayor mortalidad por diferentes causas. Por ejemplo en una revisión reciente de trabajos que estudiaban dicha relación ${ }^{4}$, recopilando información de 1,46 millones de personas adultas de raza blanca de 19 a 84 años, y controlando la influencia de otros factores de riesgo para la salud, se encontró que tanto el sobrepeso como la obesidad (y posiblemente el infrapeso), estaban relacionados con todas las causas de mortalidad. Teniendo como referencia el rango de IMC normal en adultos (20 a 24,9), se ha hallado que en las mujeres el riesgo de mortandad se incrementa en la medida en que dicho índice se aleja de esos valores, de la siguiente forma: IMC de
15 a 18,4 , incremento de un $47 \%$; IMC de 18,5 a 19,9 , incremento de un $14 \%$; IMC de 25 a 29,9: incremento de un $13 \%$; IMC de 30 a 34,9 , incremento de un $44 \%$; IMC de 35 a 39,9 , incremento de un $88 \%$; IMC de 40 a 49,9 , incremento de un $151 \%$. Los resultados obtenidos con varones son muy similares. Como puede observarse las personas con sobrepeso y obesidad, con valores de IMC superiores a 25 , incrementan poderosamente su riesgo de mortalidad con respecto a las personas en normo peso, hasta el punto que quienes tienen obesidad mórbida (IMC >40) tienen más del doble de probabilidad de morir que las que están en el rango $20-24,9$.

\section{ESTUDIOS RECIENTES SOBRE COMORBILIDAD PSICOPATOLÓGICA}

Hoy en día la obesidad no se considera un trastorno mental, estatus que no parece que se vaya a ver modificado en un futuro cercano ${ }^{5}$. Sin embargo, desde hace unas décadas se estudia la relación entre el sobrepeso o la obesidad y posibles alteraciones psicológicas concomitantes, sin que se haya llegado a un consenso sobre la existencia de un perfil psicológico específico ${ }^{6}$ en quienes los presentan, ni en el posible poder patógeno de la psicopatología en la obesidad $^{2}$. No obstante, cada vez disponemos de mayor evidencia de la posible existencia de cierta comorbilidad psicopatológica en la obesidad, incluidos algunos trabajos españoles ${ }^{7,8}$.

En la literatura científica encontramos una interesante revisión ${ }^{9}$ sobre dicha comorbilidad hasta 2007, llegando a resultados dispares y no concluyentes. Por consiguiente, este trabajo tiene por objeto actualizar la evidencia más reciente sobre esta posible comorbilidad, en particular, revisar los estudios publicados al respecto entre $2007 \mathrm{y}$ 2010. Para su realización se han utilizado las bases de datos MEDLINE y SCIELO, mediante búsquedas a través de los descriptores "obesidad", "sobrepeso", "comorbilidad" y "psicopatología". A continuación, se expone un resumen de los trabajos analizados, los cuales han sido seleccionados para garan- 
tizar la generabilidad de los datos, de modo que se han revisado estudios que emplearon sujetos procedentes de diversas poblaciones a nivel mundial, tanto de muestras clínicas como de población general, así como de estratos evolutivos diferentes. Algunas de las investigaciones incluidas exploran la relación entre variables relacionadas con la obesidad y constructos psicopatológicos, los cuales aunque no pueden considerarse propiamente trabajos sobre comorbilidad, se ha considerado que sus aportaciones sí permiten estudiarla.

En primer lugar se presentan aquellos estudios que han encontrado comorbilidad $\mathrm{y}$, posteriormente, se describen aquellos que no han hallado relación alguna entre la obesidad y distintas variables psicopatológicas, organizados a su vez en función de la metodología empleada. En la tabla 1 puede verse un resumen de la estructura de la revisión.

Tabla 1. Estructura de la revisión sobre comorbilidad y psicopatología

\begin{tabular}{|c|c|c|}
\hline & Metodología & Estudios revisados \\
\hline \multirow{4}{*}{$\begin{array}{c}\text { Trabajos que } \\
\text { confirman } \\
\text { comorbilidad } \\
\text { psicopatológica en } \\
\text { obesidad }\end{array}$} & $\begin{array}{l}\text { Encuestas } \\
\text { poblacionales de salud } \\
\text { y estudios de muestras }\end{array}$ & 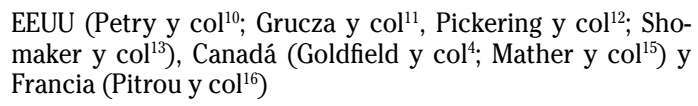 \\
\hline & $\begin{array}{l}\text { Comparación } \\
\text { intergrupos }\end{array}$ & 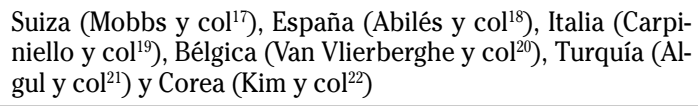 \\
\hline & $\begin{array}{l}\text { Estudios clínicos con } \\
\text { pacientes obesos o } \\
\text { candidatos a cirugía } \\
\text { biariátrica }\end{array}$ & $\begin{array}{l}\left.\text { EEUU (Lokken y col' }{ }^{23} \text {; Kalarchian y } \mathrm{col}^{24} \text {; Wadden y } \mathrm{col}^{25}\right) \text {, } \\
\text { Brasil }\left(\text { Fandiño y } \mathrm{col}^{26}\right) \text {, Francia (Isnard y } \operatorname{col}^{27} \text { ), Turquía }(\text { Ta- } \\
\text { ner y } \operatorname{col}^{28} \text { ) y España }\left(\text { Vázquez y } \operatorname{col}^{29}\right)\end{array}$ \\
\hline & Otras metodologías & $\begin{array}{l}\left.\text { Holanda (Vogelzangs y } \operatorname{col}^{30}\right) \text {, Suecia (Ernersson y } \operatorname{col}^{31} \text { ) y } \\
\left.\text { EEUU (Kasen } \operatorname{~col}^{32}\right)\end{array}$ \\
\hline $\begin{array}{l}\text { Estudios que no } \\
\text { apoyan la comorbilidad } \\
\text { entre obesidad y } \\
\text { psicopatología }\end{array}$ & $\begin{array}{l}\text { Encuestas en } \\
\text { población general y } \\
\text { estudios en pacientes } \\
\text { obesos }\end{array}$ & $\begin{array}{l}\left.\left.\text { Brasil (Papelbaum y col' }{ }^{33}\right) \text {, Australia (Goldney y } \operatorname{col}^{34}\right) \text {, y Ale- } \\
\text { mania }\left(\text { Hach y col }^{35}\right)\end{array}$ \\
\hline
\end{tabular}

\section{ESTUDIOS QUE CONFIRMAN LA COMORBILIDAD ENTRE OBESIDAD Y PSICOPATOLOGÍA}

\section{Encuestas poblacionales de salud y estudios de muestras}

Se han realizado diversas investigaciones internacionales con adultos procedentes de distintas poblaciones. Así, comenzando con macro encuestas llevadas a cabo en Estados Unidos se han encontrado los siguientes resultados: a) en $2008^{10}$, aprovechando una encuesta epidemiológica sobre alcohol y condiciones relacionadas a 141.654 personas, se encontró que aquellas obesas o extremadamente obesas, en comparación con las que no lo eran, mostraban mayores niveles de prevalencia de trastornos del ánimo, ansiedad o consumo de alcohol; b) en una encuesta similar ${ }^{11}$, publicada en 2010 , realizada en dos periodos de tiempo (19911992 y 2001-2002) entrevistando a 39.312 y 39.625 adultos respectivamente, los cuales fueron seleccionados de manera aleatoria, se encontró una fuerte asociación entre historia familiar de alcoholismo o problemas con el alcohol y la obesidad, sobre todo en mujeres; c) en un tercer informe ${ }^{12}$ publicado en 2007 a partir de datos de la encuesta nacional sobre salud elaborada también entre 2001 y 2002, se halló que el sobrepeso y la obesidad estaban relacionados (Odds ratio superiores a 1,2) con ciertos problemas de 
orden psicológico como los episodios depresivos, el trastorno de pánico, las fobias, la personalidad antisocial y el trastorno por evitación, si bien se encontraron importantes diferencias de género, dado que en hombres la relación más clara era solo entre sobrepeso y trastorno de pánico. En Estados Unidos también se han realizado estudios en población infanto-juvenil, por ejemplo, el estudio ${ }^{13}$ publicado en 2010, donde se evaluó a 367 adolescentes (12,7 años de edad media) en quienes observaron que aquellos con comportamiento compulsivo con respecto a la comida tenían significativamente mayor tendencia hacia los trastornos alimentarios, así como más síntomas depresivos y de ansiedad, que aquellos que no tenían dicho comportamiento compulsivo.

En otros países, utilizando también la metodología de encuestas se ha encontrado comorbilidad entre obesidad y psicopatología. En Canadá destacan los siguientes dos trabajos: en el primero, publicado en $2010^{14}$, en el que se encuestó a 1.490 adolescentes, se comprobó que aquellos que eran obesos, en comparación con los adolescentes con peso bajo o normal, tenían mayor insatisfacción corporal y mayor sintomatología depresiva, en particular, niveles más elevados de anhedonia, autoestima negativa y depresión general; en el segundo, publicado en $2009^{15}$ y relativo a una encuesta de salud comunitaria realizada a 36.984 personas mayores de 15 años siguiendo los criterios del Diagnostic and Statistical Manual of Mental Disorders cuarta edición (DSMIV), se comprobó que la obesidad estaba asociada a una mayor probabilidad de padecer diferentes trastornos mentales como depresión, manía, ataques de pánico, fobia social y agorafobia sin trastorno de pánico, así como ideación y tentativa suicidas.

Por otro lado, en Francia en $2010^{16}$ se publicó una encuesta realizada a 1.030 niños de 6 a 11 años y a sus familiares, de la que se concluyó que el exceso de peso estaba asociado no solo a ciertas variables psicosociales, como bajos ingresos mensuales, sino también con variables psicopatológicas como mayores niveles de ansiedad generalizada, trastornos de conducta infor- mados por los padres, problemas emocionales y dificultades con los compañeros.

\section{Comparación intergrupos}

Otra metodología empleada habitualmente en los estudios sobre comorbilidad es la comparación entre grupos, en la cual se selecciona un grupo de personas con alto IMC, o pacientes procedentes de unidades de tratamiento de la obesidad, y se les compara con otro grupo demográficamente similar, pero que no presenta sobrepeso ni obesidad. De entre las investigaciones que han hallado comorbilidad empleando dicha metodología, pueden destacarse los que se resumen a continuación, llevados a cabo en diversos países.

En 2010 se publica en Suiza ${ }^{17}$ una comparación entre 47 personas con normopeso y 47 personas con sobrepeso u obesidad, concluyéndose que estas últimas presentan mayor alteración en diferentes facetas de la impulsividad, tales como menores niveles en el control de la urgencia, perseverancia y sensibilidad a la recompensa.

Una investigación en España ${ }^{18}$, publicada en 2010, que comparó 50 pacientes obesos candidatos a cirugía bariátrica con 24 personas voluntarias de peso normal, encontró que los pacientes obesos mostraban niveles más altos de estrés, ansiedad, depresión y ansia por la comida, así como niveles más bajos de autoestima, síntomas de trastorno del comportamiento alimentario y una peor calidad de vida, en comparación con los sujetos controles con normo-peso. En Italia ${ }^{19}$, se publica en 2009 un estudio que empleó 293 pacientes con obesidad y 293 personas control en normopeso, determinándose que la obesidad se asocia de manera significativa con un mayor riesgo de comorbilidad psiquiátrica y peor calidad de la vida, teniendo en cuenta los criterios DSM-IV y OMS respectivamente. También ese año, en Bélgica $^{20}$, compararon 155 adolescentes con sobrepeso (media de edad 13,7 años) con 73 que no lo presentaban y comprobaron que el 37,5\% de los participantes en el grupo con sobrepeso cumplían los criterios DSMIV para al menos un trastorno mental, sien- 
do los trastornos más comunes los de tipo ansioso, frente al 23,29\% del primer grupo. En Turquía ${ }^{21}$ se publicó en 2009 un estudio comparativo entre 124 pacientes obesos y 106 sujetos control sanos, los cuales fueron evaluados mediante instrumentos como el Symptom Checklist-90-Revised (SCL-90-R) y el Short Form 36 Health Survey Questionnaire (SF-36). Los resultados constataban que el grupo con mayor obesidad (Clase II) poseía un peor estatus psicológico, calidad de vida y calidad de sueño que el grupo control. Por último, en Corea $^{22}$, empleando esta misma metodología, se publicó en 2007 una encuesta a 534 pacientes mujeres con sobrepeso u obesidad. Aquellas pacientes obesas, en comparación con 398 mujeres sanas procedentes de un grupo control, y aquellas que presentaban sobrepeso, tenían niveles más altos de depresión, mayor número de actitudes alteradas respecto al comportamiento alimentario y peor calidad de vida.

\section{Estudios clínicos con pacientes obesos o candidatos a cirugía bariátrica}

Una tercera estrategia metodológica empleada en diferentes trabajos consiste en analizar la posible sintomatología psicopatológica en pacientes obesos, o pacientes candidatos a cirugía bariátrica. Así, en Estados Unidos se publican en $2010^{23}$ los resultados de una evaluación psicológica realizada a 169 pacientes con obesidad mórbida candidatos a cirugía bariátrica, encontrándose que éstos muestran ciertos déficits cognitivos, en particular, en sus funciones ejecutivas, tales como la planificación o resolución de problemas, en referencia a la población base, si bien no se halló singularidad psicopatológica frente a otros pacientes sometidos a cirugía. Otra investigación realizada anteriormente en el mismo país y publicada en $2007^{24}$, empleando 288 pacientes de cirugía bariátrica, halló que cerca del $66 \%$ de ellos había tenido algún episodio de trastorno psicopatológico (Eje I del DSM-IV) a lo largo de su vida; por otro lado, el $38 \%$ cumplía los criterios para dicho diagnóstico en el momento en el que se llevó a cabo el estudio. La mani- festación psicopatológica estaba asociada al nivel de IMC de los sujetos. El mismo año y también en dicho país, se publica una revisión ${ }^{25}$ bibliográfica junto a los datos de 2.500 pacientes candidatos a cirugía bariátrica, analizando la existencia de mayor riesgo psicosocial en dichos sujetos.

En Brasil en $2010^{26}$ se publica un estudio con 212 mujeres obesas quienes buscaban tratamiento, y hallaron que además de obesidad, el 26,6\% tienen un trastorno por atracón, y que éstas muestran mayores alteraciones de salud y sintomatología psicopatológica, como depresión, que quienes eran obesas sin trastorno por atracón.

En Francia se publicó un trabajo en $2010^{27}$, donde se estudió a 115 adolescentes de 12 a 17 años, pacientes obesos, a los que se les evaluó posible sintomatología bulímica, depresiva y ansiosa, encontrando relaciones entre dichas variables, lo que llevó a los autores a recomendar la inclusión de la evaluación psiquiátrica en los casos de obesidad en adolescentes.

En $2009^{28}$ se publica una investigación llevada a cabo en Turquía donde se investiga a 54 pacientes pediátricos obesos hallándose que el 50\% de la muestra mostraba síntomas psicopatológicos concomitantes a la obesidad; dichos sujetos, a su vez, cumplían en menor medida los tratamientos indicados para combatir dicha obesidad. Así mismo, en España, un trabajo publicado en $2008^{29}$, en el cual se había recogido la valoración sobre salud mental de 333 pacientes enviados a un servicio de Endocrinología y Nutrición por motivos de sobrepeso u obesidad, arrojó como resultado la presencia de sintomatología psicopatológica en el $47,1 \%$ de los casos evaluados a través de cuestionarios como el General Health Questionnaire (GHQ) o inventarios específicos de trastornos de la conducta alimentaria, así como una mayor prevalencia patológica en el sexo femenino.

\section{Otras metodologías}

Por último, existen otro tipo de estrategias metodológicas a través de las cuales también se ha encontrado comorbilidad. 
Una investigación de carácter prospectivo sobre salud, envejecimiento y composición corporal publicada en $2010^{30}$ en Holanda con 2.547 personas de edad avanzada (7079 años), reveló que la obesidad, en particular -tener mucha grasa visceral- aumenta significativamente el riesgo de aparición de síntomas depresivos en los hombres.

En $2010^{31}$ se publicó un estudio en Suecia que empleaba una metodología cuasi-experimental donde se comparaba a un grupo de 18 adultos a los que se les instruyó para que llevaran a cabo una sobreingesta de calorías y se comportaran sedentariamente durante cuatro semanas, con un grupo control de 18 adultos a los que se les solicitó que no modificaran su dieta ni actividad física. El grupo experimental sufrió un empeoramiento de su calidad de vida asociada a la salud con respecto al grupo control, manifestándose en un aumento de peso corporal y peores puntuaciones de salud mental en el Short Form 36 Health Survey Questionnaire (SF-36), así como mayores niveles de sintomatología depresiva evaluada mediante cuestionarios específicos. Un último estudio destacable en este apartado es aquel de carácter longitudinal publicado en $2007^{32}$, pero llevado a cabo a lo largo de tres décadas a 544 mujeres en EEUU. Éste concluyó que los niveles más altos de IMC (a partir de 30) estaban claramente asociados con un mayor riesgo de padecer trastorno por ansiedad generalizada y trastorno depresivo mayor.

\section{ESTUDIOS QUE NO APOYAN LA COMORBILIDAD ENTRE OBESIDAD Y PSICOPATOLOGÍA}

Sin embargo, no todos los estudios revisados en este periodo temporal encontraron comorbilidad. Por ejemplo, en una investigación publicada en $2010^{33}$ y realizada en Brasil con 217 mujeres obesas evaluadas por posible psicopatología mediante cuestionarios como el Beck Depression Inventory (BDI) y el Symptom Checklist-90 (SCL-90), se encontró que su grado de obesidad no correlacionaba con variables psicopatológicas habituales como ansiedad o depresión; únicamente se encontró una relación positiva y significativa con la presencia de somatización.

Así mismo, en un estudio realizado en Australia en $2009^{34}$ donde se entrevistó a una muestra representativa de la población acerca de su IMC, su salud mental e ideación suicida, a través de 3.034 entrevistas cara a cara y 30.214 mediante un sistema telefónico automático, se concluyó que no existía correlación entre un mayor IMC y mayores problemas de salud mental o ideación de suicidio. Por el contrario, los niveles más altos de IMC parecían ser factor de protección de dicha sintomatología.

Por último, se puede mencionar una encuesta de salud llevaba a cabo en Alemania a 4.181 personas adultas y publicada en $2007^{35}$. En ella se observa que si bien la obesidad está relacionada con peores condiciones psicosociales, como un menor estatus económico, no aparecen relaciones significativas entre ésta y una peor salud mental.

\section{ANÁLISIS DE LAS INVESTIGACIONES}

El análisis de las investigaciones anteriormente mencionadas proporciona diversas consideraciones. Por una parte, la mayoría de los trabajos hallados en la literatura científica apuntan la existencia de cierta comorbilidad psicopatológica en obesidad, a pesar de que ciertos estudios más aislados no la encuentran, e incluso en alguno muy concreto ${ }^{34}$ se observa un perfil psicológico más adaptado en las personas con mayor IMC. El hallazgo de resultados contradictorios entre investigaciones nos permite dudar sobre si la metodología empleada es la más adecuada en cada uno de los trabajos. Si consideramos que no se produce dicha problemática metodológica, podemos estimar que las diferencias culturales son las que matizan la posible existencia de comorbilidad, el grado en el que se produce la misma y la psicopatología implicada. Es necesario tener en cuenta que en algunos casos la variable utilizada es el IMC, sin comprobar que en la muestra hubiera claramente obesidad; en otros casos sí se examina a sujetos clasificados como obesos. Estas discrepancias en la selección 
y evaluación de los sujetos podrían matizar los resultados, y quizás dar mayor credibilidad a los que utilizan la segunda metodología basada en el diagnóstico clínico.

Donde se encuentra una mayor presencia de alteraciones psicológicas de diversa índole es en los estudios cuyos participantes son pacientes obesos que forman parte de programas de cirugía bariátrica, que por otra parte era esperable, pues la obesidad mórbida puede estar asociada a problemas previos de orden psicosocial o, teniendo en cuenta cómo se manifiesta dicho tipo de obesidad, puede llegar a causarlos. En este sentido, diversos trabajos, alguno de ellos realizados en España ${ }^{36}$, han encontrado cómo el perfil psicopatológico de pacientes sometidos a cirugía bariátrica mejora tras el tratamiento quirúrgico.

A pesar de la aparente contundencia en los resultados de ciertas investigaciones, señalando la presencia de comorbilidad, por el momento, éstos deben ser tomados como simples indicadores de una relación, no como demostraciones causales entre la obesidad y las distintas alteraciones psicológicas analizadas. La metodología correlacional de la mayoría de los trabajos, ya que son raros los estudios que emplean metodología experimental o cuasi-experimental, no permite establecer conclusiones más contundentes y específicas. En este sentido, conviene recordar que la existencia de una comorbilidad psicopatológica en obesidad se ha entendido desde dos perspectivas. Por una parte, como un posible factor explicativo, ya que puede ser entendible que una persona que tiene un trastorno depresivo intente compensar parte de la sintomatología con el placer de comer y que la tendencia al aislamiento característica de esta patología facilite un sedentarismo, todo lo cual favorezca, a su vez, un incremento de peso. Pero, también puede entenderse en la dirección contraria, que una persona sin alteración psicológica previa, que sufre obesidad debido a sus malos hábitos alimenticios o a una predisposición genética, puede generarle cierto rechazo social o la disminución de sus actividades cotidianas de interacción, de modo que termine por desarrollar sintomatología depresiva.

Con respecto a las diferencias de género, de la revisión no pueden extraerse evidencias rotundas, pues algunos estudios ${ }^{29}$ encuentran mayor comorbilidad en mujeres mientras otros han detectado dicha tendencia en los varones ${ }^{30}$.

\section{CONCLUSIONES Y RECOMENDACIONES}

Teniendo en cuenta las anteriores matizaciones y observaciones se ha llegado a las siguientes conclusiones, sugiriéndose las correspondientes recomendaciones:

1. Aunque existen trabajos con resultados contradictorios, la literatura científica revisada en el periodo temporal objeto de este estudio, encuentra mayoritariamente algún tipo de comorbilidad psicopatológica en las personas con sobrepeso u obesidad. La investigación en España al respecto sigue siendo reducida y centrada en población clínica obesa, sin disponer aún de estudios con población general y diferente rango de edad, los cuales consideramos serían necesarios, recomendación que también proponen otros autores ${ }^{9}$.

2. La comorbilidad psicopatológica más prevalente parece estar relacionada con trastornos del estado ánimo, la ansiedad, el consumo de sustancias y los trastornos del comportamiento alimentario. Este sesgo puede deberse a que las investigaciones revisadas estaban en gran medida dirigidas a encontrar evidencias de esos trastornos y no otros, por lo que no puede concluirse que se trate de la única comorbilidad posible respecto de la obesidad. Por otro lado, algunos autores ${ }^{2}$ abundan en la explicación de por qué la obesidad está asociada a trastornos psicológicos como los mencionados, valorando cuándo la obesidad puede ser causa o consecuencia de la psicopatología.

3. La alteración psicopatológica concomitante en los pacientes con obesidad puede ser la causa explicativa más importante $^{37}$ del empeoramiento de su calidad de vida asociada a la salud y, particularmente, en pacientes sometidos a cirugía bariátri- 
ca, puede constituir un factor de riesgo de peor recuperación ${ }^{38}$ postoperatoria, por lo que en estos últimos pacientes el seguimiento psicológico y/o psiquiátrico se hace imprescindible.

4. Dadas las anteriores conclusiones se considera necesario incluir una evaluación de carácter psicopatológico en los programas de intervención de pacientes con obesidad, y valorar consecuentemente si debe implementarse un tratamiento de orden psicológico y/o psiquiátrico según el diagnóstico resultante, sugerencia que es acorde con la propuesta de diversos autores $^{19,21,22,39-41}$.

5. Conviene que los psicólogos clínicos y los psiquiatras adquieran formación específica sobre la obesidad, sus manifestaciones y los protocolos de intervención óptimos para tratar a estos pacientes desde todas las perspectivas, sugerencia que también realizan otros autores ${ }^{42,43}$.

\section{BIBLIOGRAFÍA}

1. Moreno B, Monereo S. Álvarez J. Obesidad. La epidemia del siglo XXI. Madrid: Díaz de Santos, 2000.

2. Chinchllla A. Obesidad y psiquiatría. Barcelona: Masson; 2005.

3. Organización Mundial de la Salud-OMS. Obesidad y sobrepeso [sede Web]. [acceso 01 de enero de 2011]. Disponible en: http:// www.who.int/mediacentre/factsheets/fs311/ es/index.html

4. Berrington de Gonzalez A, Hartge P, Cerhan JR, Fuint AJ, Hannan L, MacInnis RJ et al. Bodymass index and mortality among 1.46 million white adults. Health Qual Life Outcomes 2010; 23: 8-90.

5. Marcus MD, Wildes JE. Obesity: is it a mental disorder? Int J Eat Disord 2009; 42: 739-753.

6. Luna I. Psicopatología y obesidad. Avan Psiq Bio 2007; 8: 58-84.

7. Arias F, SÁnchez S, Gorgojo JJ, Almódovar F, FerNÁNDEZ S, LLORENTE F. Diferencias clínicas entre pacientes obesos mórbidos con y sin atracones. Actas Esp Psiquiatr 2006; 34: 362-370.

8. Delgado C, Morales MJ, Maruri I, Rodríguez, Benavente JL, Núnez S. Conductas alimentarias, actitudes hacia el cuerpo y psicopatología en obesidad mórbida. Actas Esp Psiquiatr 2002; 30: 376-381.
9. Lово A, Lово E. Morbilidad psíquica en pacientes obesos. Med Clin (Barc) 2008; 130: 54-56.

10. Petry NM, Barry D, Pietrzak RH, Wagner JA. Overweight and obesity are associated with psychiatric disorders: results from the National Epidemiologic Survey on Alcohol and Related Conditions. Psychosom Med 2008; 70: 288-297.

11. Grucza RA, Krueger RF, Racette SB, Norberg KE, HIPP PR, BIERUT LJ.The emerging link between alcoholism risk and obesity in the United States. Arch Gen Psychiatry 2010; 67: 1301-1308.

12. Pickering RP, Grant BF, Chou SP, Compton WM. Are overweight, obesity, and extreme obesity associated with psychopathology? Results from the national epidemiologic survey on alcohol and related conditions. J Clin Psychiatry 2007; 68: 998-1009.

13. Shomaker LB, Tanofsky-KrafF M, Elliott C, WoLKoff LE, Columbo KM, Ranzenhofer LM et al. Salience of loss of control for pediatric binge episodes: does size really matter? Int J Eat Disord 2010; 43: 707-716.

14. Goldfield GS, Moore C, Henderson K, Buchiolz A, Obeid N, Flament MF. Body dissatisfaction, dietary restraint, depression, and weight status in adolescents. J Sch Health 2010; 80: 186192.

15. Mather AA, Cox BJ, Enns MW, Sareen J. Associations of obesity with psychiatric disorders and suicidal behaviors in a nationally representative sample. J Psychosom Res 2009; 66: 277-285.

16. Pitrou I, Shojaei T, Wazana A, Gilbert F, KovessMASFÉtY V. Child overweight, associated psychopathology, and social functioning: a French school-based survey in 6- to 11-yearold children. Obesity 2010; 18: 809-817.

17. Mobbs O, Crépin C, Thí́RY C, Golay A, VAn der LINDEN M. Obesity and the four facets of impulsivity. Patient Educ Couns 2010; 79: 372377.

18. Abilés V, Rodríguez-Ruiz S, Abilés J, Mellado C, García A, Pérez de la Cruz A et al. Psychological characteristics of morbidly obese candidates for bariatric surgery. Obes Surg 2010; 20: 161-167.

19. Carpiniello B, Pinna F, Pillai G, Nonnol V, PisaNo E, Corrias S et al. Psychiatric comorbidity and quality of life in obese patients. Results from a case-control study. Int J Psychiatry Med 2009; 39: 63-78.

20. Van Vlierberghe L, Braet C, Goossens L, Mels S. Psychiatric disorders and symptom severity 
in referred versus non-referred overweight children and adolescents. Eur Child Adolesc Psychiatry 2009; 18: 164-173.

21. Algul A, Ates MA, Semiz UB, Basoglu C, Ebrinc $\mathrm{S}$, GeCICI $\mathrm{O}$ et al. Evaluation of general psychopathology, subjective sleep quality, and health-related quality of life in patients with obesity. Int J Psychiatry Med 2009; 39: 297312.

22. Кім JY, Он DJ, Yoon TY, Сног JM, Сное BK. The impacts of obesity on psychological wellbeing: a cross-sectional study about depressive mood and quality of life. J Prev Med Public Health 2007; 40: 191-195.

23. LoKken KL, Boeka AG, Yellumahanthi K, Wesley M, Clements RH. Cognitive performance of morbidly obese patients seeking bariatric surgery. Am Surg 2010; 76: 55-59.

24. Kalarchian MA, Marcus MD, Levine MD, Courcoulas AP, Pilkonis PA, Ringham RM et al. Psychiatric disorders among bariatric surgery candidates: relationship to obesity and functional health status. Am J Psychiatry 2007; 164: 328-334.

25. Wadden TA, Sarwer DB, Fabricatore AN, Jones L, Stack R, Williams NS. Psychosocial and behavioral status of patients undergoing bariatric surgery: what to expect before and after surgery. Med Clin North Am 2007; 91: 451-469.

26. Fandiño J, Moreira RO, Preissler C, Gaya CW, Papelbaum M, CoutinHo WF et al. Impact of binge eating disorder in the psychopathological profile of obese women. Compr Psychiatry 2010; 51: 110-114.

27. Isnard P, Quantin L, Cortese S, Falissard B, MusHer-EizenMan D, Guedeney et al. Bulimic behaviours and psychopathology in obese adolescents and in their parents. Int $\mathrm{J}$ Pediatr Obes 2010; 5: 474-482.

28. Taner Y, Törel-Ergür A, Bahçivan G, Gürdag M. Psychopathology and its effect on treatment compliance in pediatric obesity patients. Turk J Pediatr 2009; 51: 466-471.

29. Vázquez C, Alcaraz F, Balsa Ja, Zamarrón I, Arrieta F, Botella-Carretero JI. Prevalence of psychiatric cases in overweight or obese patients attended in a hospital outpatient clinic. Med Clin (Barc) 2008; 130: 41-46.

30. Vogelzangs N, Kritchevsky SB, Beekman AT, Brenes GA, Newman AB, SatTerfield $S$ et al. Obesity and onset of significant depressive symptoms: results from a prospective community-based cohort study of older men and women. J Clin Psychiatry 2010; 71: 391-399.

31. Ernersson A, Frisman GH, Sepa Frostell A, NysTRÖM FH, LINDSTRÖM T. An obesity provoking behaviour negatively influences young normal weight subjects' health related quality of life and causes depressive symptoms. Eat Behav 2010; 11: 247-252.

32. Kasen S, Cohen P, Chen H, Must A. Obesity and psychopathology in women: a three decade prospective study. Int J Obes (Lond) 2008; 32: 558-566.

33. Papelbaum M, Moreira Ro, Gaya CW, Preissler C, Coutinho WF. Impact of body mass index on the psychopathological profile of obese women. Rev Bras Psiquiatr 2010; 32: 42-46.

34. Goldney RD, Dunn KI, Air TM, Dal Grande E, TAYLOR AW. Relationships between body mass index, mental health, and suicidal ideation: population perspective using two methods. Aust N Z J Psychiatry 2009; 43: 652-658.

35. Hach I, Ruhl UE, Klose M, Klotsche J, Kirch $\mathrm{W}$, JACOBI F. Obesity and the risk for mental disorders in a representative German adult sample. Eur J Public Health 2007; 17: 297-305.

36. Zaldínar S, Arias F, Gorgojo JJ, Romero S. Evolución de alteraciones psicopatológicas en pacientes con obesidad mórbida tras cirugía bariátrica. Med Clin (Barc) 2009; 133: 206-212.

37. Mannucci E, Petroni ML, Villanova N, Rotella CM, APOLONE G, Marchesini G; QUOVADIS Study Group. Clinical and psychological correlates of health-related quality of life in obese patients. Health Qual Life Outcomes 2010; 23; 8-90.

38. Botella F, Alfaro JJ, Pacheco E, lomas A, Salas MA, GARCía A et al. Influencia de la enfermedad psiquiátrica previa en la evolución de los pacientes sometidos a cirugía bariátrica. Endocrinol Nutr 2010; 57: 9-15.

39. Pull CB. Current psychological assessment practices in obesity surgery programs: what to assess and why. Curr Opin Psychiatry 2010; 23: 30-36.

40. DRUKker M, Wojciechowski F, Feron FJ, Mengelers $\mathrm{R}, \mathrm{VAN}$ Os J. A community study of psychosocial functioning and weight in young children and adolescents. Int J Pediatr Obes 2009; 4: 91-97.

41. Montero J, Cúneo A, Facchini M, Bressan J. Tratamiento integral de la obesidad y su prevención. An Sist Sanit Navar 2002; 25 (Supl 1): 175-186.

42. Lichwala-Zyla C, Price JH, Dake JA, Jordan T, PRICE JA. Psychiatrists' perceptions and practices in treating patients' obesity. Acad Psychiatry 2009; 33: 370-376.

43. Bean MK, Stewart K, Olbrisch ME. Obesity in America: implications for clinical and health psychologists. J Clin Psychol Med Settings 2008; 15: 214-224. 
